\title{
A Practice Facilitation and Academic Detailing Intervention Can Improve Cancer Screening Rates in Primary Care Safety Net Clinics
}

\author{
Emily M. Mader, MPH, MPP, Chester H. Fox, MD, John W. Epling, MD, MSEd, \\ Gary J. Noronha, MD, Carlos M. Swanger, MD, Angela M. Wisniewski, PharmD, \\ Karen Vitale, MSEd, Amanda L. Norton, MSW, and Christopher P. Morley, PhD
}

Background: Despite the current evidence of preventive screening effectiveness, rates of breast, cervical, and colorectal cancer in the United States fall below national targets. We evaluated the efficacy and feasibility of combining practice facilitation and academic detailing quality improvement (QI) strategies to help primary care practices increase breast, cervical, and colorectal cancer screening among patients.

Methods: Practices received a 1-hour academic detailing session addressing current cancer screening guidelines and best practices, followed by 6 months of practice facilitation to implement evidence-based interventions aimed at increasing patient screening. One-way repeated measures analysis of variance compared screening rates before and after the intervention, provider surveys, and TRANSLATE model scores. Qualitative data were gathered via participant focus groups and interviews.

Results: Twenty-three practices enrolled in the project: 4 federally qualified health centers, 10 practices affiliated with larger health systems, 4 physician-owned practices, 4 university hospital clinics, and 1 nonprofit clinic. Average screening rates for breast cancer increased by $13 \%(P=.001)$, and rates for colorectal cancer increased by 5.6\% $(P=.001)$. Practices implemented a mix of electronic health record data cleaning workflows, provider audits and feedback, reminder systems streamlining, and patient education and outreach interventions. Practice facilitators assisted practices in tailoring interventions to practice-specific priorities and constraints and in connecting with community resources. Practices with resource constraints benefited from the engagement of all levels of staff in the quality improvement processes and from team-based adaptations to office workflows and policies. Many practices aligned quality improvement interventions in this project with patient-centered medical home and other regulatory reporting targets.

Conclusions: Combining practice facilitation and academic detailing is 1 method through which primary care practices can achieve systems-level changes to better manage patient population health. (J Am Board Fam Med 2016;29:533-542.)

Keywords: Analysis of Variance, Breast Cancer, Cancer Prevention \& Control, Cervical Cancer, Colorectal Cancer, Early Detection of Cancer, Focus Groups, Primary Health Care, Quality, Improvement, Reminder Systems, Surveys \& Questionnaires, Workflow

Screening for breast, cervical, and colorectal cancers can detect disease at an early stage, when it is most amenable to treatment, effectively reducing

This article was externally peer reviewed.

Submitted 31 March 2016; revised 13 June 2016; accepted 20 June 2016.

From the Department of Family Medicine, State University of New York Upstate Medical University, Syracuse (EMM, JWE, ALN, CPM); the Department of Family Medicine, State University of New York at Buffalo (CHF, AMW); the Department of Public Health \& Preventive Medicine, State University of New York Upstate Medical University, Syracuse (JWE, CPM); the Center for Primary mortality and morbidity. ${ }^{1}$ The colorectal cancer (CRC) screening methods of high-sensitivity stool

Care, University of Rochester School of Medicine and Dentistry, Rochester, NY (GJN, CMS); the Center for Community Health, University of Rochester School of Medicine and Dentistry, Rochester (KV); and the Department of Psychiatry \& Behavioral Sciences, State University of New York Upstate Medical University, Syracuse (CPM).

Funding: This publication was supported by grant or cooperative agreement DP2029 and DP3879 between the Centers for Disease Control and Prevention (CDC) and the New York State Department of Health and funded by the CDC. 
tests, flexible sigmoidoscopy, and colonoscopy; breast cancer screening through mammography; and cervical cancer screening through Papanicolaou tests have been identified by the US Preventive Services Task Force (USPSTF) as effective preventive measures for the early identification of CRC, breast cancer, and cervical cancer. ${ }^{2}$ However, rates of screening for these cancers remain below the national Healthy People 2020 targets in the United States. ${ }^{3}$ In addition, large segments of the population (including the uninsured, rural residents, Hispanics/Latinos, African Americans, Native Americans, and those with low education levels and low socioeconomic status) have been shown to be significantly less likely to receive appropriate cancer screening and will likely experience a higher burden of cancer because of suboptimal screening. ${ }^{4-9}$

A variety of factors present challenges to the effective provision of cancer screening in primary care, including numerous guideline recommendations, patient reluctance or refusal, and resource constraints. ${ }^{10-13}$ Primary care practices can benefit from interventions and techniques to address barriers to the effective provision of population health maintenance services, including cancer screening. One potential intervention to achieve this goal is the use of practice facilitation and academic detailing.

Practice facilitation involves the work of trained quality improvement (QI) professionals who assist primary care practices in research and QI activities. ${ }^{14-16}$ Assistance includes data collection, feedback on provider and practice performance, and the facilitation of systems-level changes to improve practice processes. Academic detailing is modeled based on the communication approach of pharmaceutical industry detailers and involves trained experts visiting health care professionals in their own setting to provide tailored education on specific health topics and evidence-based guidance on best practices. ${ }^{17-19}$ The goal of combined practice facil-

Conflict of interest: none declared.

Disclaimer: The contents of this article are solely the responsibility of the authors and do not necessarily represent the official views of the CDC, the US Department of Health and Human Services, or the New York State Department of Health.

Corresponding author: Christopher P. Morley, PhD, Department of Family Medicine, SUNY Upstate Medical University College of Medicine, 750 E. Adams St, Syracuse, NY 13210 (E-mail: morleycp@upstate.edu). itation and academic detailing is to help primary care practices align their work with evidence-based best practices to improve patient care and outcomes. $^{14,20}$

The purpose of the project detailed in this article was to evaluate the efficacy and feasibility of combining practice facilitation and academic detailing to help primary care practices increase patient breast cancer, cervical cancer, and CRC screening in 3 regional practice-based research networks (PBRNs) in Central and Western New York: the Studying-Acting-Learning-Teaching Network (Syracuse, NY), the Upstate New York PBRN (Buffalo, NY), and the Greater Rochester PBRN (Rochester, NY). This evaluation investigated the impact of this grant-funded intervention on cancer screening rates, as well as how components of the intervention were implemented across varying practice structures.

\section{Methods \\ Practice Facilitation and Academic Detailing Intervention}

Primary care practices were recruited for enrollment based on their capacity to affect a high percentage of patients among the following populations: racial/ethnic minorities, those with low socioeconomic status, the uninsured, those from geographically isolated/rural locations, and Medicaid-eligible populations. Twenty-seven practices were approached for enrollment, all of which had established relationships with 1 of the 3 participating regional PBRNs.

Physicians, nurses, and other care providers at each practice received a 1-hour, continuing medical education-accredited academic detailing session (ADS) presented by a primary care physician with expertise in cancer prevention recommendations. After completing the ADS, enrolled practices received a minimum of 6 months of practice facilitation services provided by 1 of 4 trained practice facilitators (PFs) from 2014 to 2015. The intervention period was limited to 6 months because of the 1-year funding cycle of the project. All PFs had formal training in QI coaching in the health care setting, with a minimum of 2 years' experience in the field. The focus of the practice facilitation intervention targeted evidence-based strategies to increase breast cancer, cervical cancer, and CRC screening (identified through the Centers for Dis- 
ease Control and Prevention's Community Guide to Preventive Services ${ }^{21}$ ), as well as improvements related to electronic health record (EHR) data.

PFs met with key personnel at each practice, including medical directors, practice managers, and other clinical staff, to review current office workflows and policies, as well as clinic performance in cancer screening. Each practice was afforded flexibility in determining their specific interventions to accommodate differences in practice size, location, administrative structure, and performance priorities. Selected interventions were required to be considered evidence-based, as determined by the benchmarks established in the Centers for Disease Control and Prevention's Community Guide to Preventive Services. The project team leadership and the program officer of the funder jointly reviewed all interventions to ensure each met evidence-based criteria.

\section{Data Collection and Analysis}

\section{Changes in Screening Rates}

Practices reported the aggregated number of all current patients within the eligible screening population (denominator) and the number of current patients who had received appropriate screening for breast cancer, cervical cancer, or CRC (numerator), according to the most recent screening guidelines from the USPSTF and/or the American Cancer Society, at both the initiation and conclusion of the 6-month practice facilitation period. Practices chose to follow the guideline of their choice based on provider preferences and reporting procedures in effect at the time of project initiation. These numbers were used to calculate a practicelevel measure of the proportion of patients who had received appropriate screening, as documented in the practice EHR, for each practice both before and after the intervention. The criteria used to define current patients generally consisted of patients seen at least once within the past 1 to 3 years, depending on practice protocols. Comparisons between cancer screening rates before and after the intervention were evaluated using 1-way repeated measures analysis of variance (ANOVA).

\section{Changes in Practice Staff Attitudes and Experiences}

Surveys were administered to clinical care team staff (physicians, nurses, care coordinators) and office administrative staff working at each practice; the PFs administered the surveys directly following the ADS and again at the close of the 6-month practice facilitation period. The surveys collected anonymous demographic information and responses to questions regarding respondent attitudes and experiences (using a 5-point Likert scale). The language and question items used in the surveys were adapted from the National Cancer Institute's Survey of Primary Care Physicians' Recommendations \& Practice for Breast, Cervical, Colorectal, \& Lung Cancer Screening 22 and $\mathrm{Na}$ tional Surveys of Colorectal Cancer Screening Policies \& Practices, ${ }^{23}$ as well as surveys developed by Houser et $\mathrm{al}^{24}$ and the Michigan Department of Community Health ${ }^{25}$; the survey language was altered to adapt questions to a 5-point Likert scale structure. Unique identifiers were used to link the survey information from before and after the intervention; mean scores on responses were compared between the 2 measurement periods through paired-samples $t$ tests.

Focus groups were conducted at each of the participating practices to solicit feedback on barriers to cancer screening, experiences working with PFs in the intervention, and suggestions for intervention improvement; key informant interviews were conducted when focus groups could not be convened because of practice staff constraints. The participants targeted for inclusion were those identified by the PFs at each practice site as having been most directly involved in the implementation of the project, and they included both clinical providers and administrative staff. The focus groups were hosted at the practice offices, whereas interviews were conducted via a telephone conference call; all focus groups and interviews were conducted by a member of the project leadership team trained in qualitative interviewing techniques. PFs were excluded from any focus group and interview activities pertaining to their assigned practices to reduce bias in participant responses.

All focus groups and interviews were audio-recorded and transcribed verbatim for analysis; no names or other personally identifiable information were recorded in the transcripts. Two members of the project team jointly conducted a thematic content analysis of the transcripts, developing a set of codes based on a list of areas of interest determined a priori: barriers to increasing cancer screening, factors important to the working relationship with the $\mathrm{PF}$, factors important for sustainable change, and feedback on project processes. Discrepancies 
between the coding schemes were resolved verbally, and the finalized themes and concepts were reviewed by the larger team.

\section{Practice Readiness for Transformation}

The TRANSLATE model was used to assess the intervention's impact on key elements of practice transformation. The TRANSLATE model is an assessment tool that measures elements of practice improvement and has been used by researchers conducting similar interventions focused on diabetes care and chronic kidney disease management. ${ }^{26,27}$ TRANSLATE stands for "set your Target, use Registry and Reminder systems, get Administrative buy-in, Network information systems, Site coordination, Local Physician Champion, Audit and feedback, Team approach, and Education." 27 Each practice was rated twice by their assigned PF for each of these 9 elementsonce after the initial visit and once at the conclusion of project activities. Each TRANSLATE category was rated on a scale of 1 to 4 , with 1 signifying no accomplishment and 4 signifying the highest accomplishment.

PFs received training on the use of the TRANSLATE model before engaging with their practices, including an overview of definitions and a review of practice characteristics meriting each score level. The TRANSLATE assessments were used by PFs as a guide for the work completed with each practice and as a measurement tool for systems-level change within each practice at the conclusion of the project. Practice-level changes in TRANSLATE element scores before and after the intervention and the influence of PF practice groupings were evaluated through a 1-way mixed ANOVA. Spearman correlations were calculated to assess the relationship between both the cancer screening rates and TRANSLATE element scores after the intervention.

\section{Human Subjects Protection}

The Institutional Review Board of State University of New York Upstate Medical University determined that this QI project did not meet the definition of human subjects research. All individuals and practices participating in the project were provided information regarding the voluntary nature of participation, and no individually identifiable information was collected.

\section{Results}

\section{Intervention Activities}

A total of 23 of the 27 primary care practices approached were enrolled in this project. Table 1 summarizes practice characteristics. The majority of practices ${ }^{16}$ were located in urban areas, and 2 of these practices hosted primary care resident physicians. Four separate EHR systems were used across the 23 practices. A total of 210 clinical providers attended the ADS at their respective practices; perpractice attendance can be found in Table 1 . The largest group of ADS attendees (37\%) was physicians, followed by nurse practitioners, physician assistants, nursing staff, and administrative staff.

The cumulative time devoted to practice facilitation activities by the PFs was approximately 889 hours. Each facilitator was assigned either 5 or 6 practices. Practice facilitation activities included efforts to address documentation procedures for screening referral and completion within practice EHR systems, provider feedback and assessment activities, streamlining of provider reminder systems and office workflows, and patient education and outreach interventions.

\section{Cancer Screening Rates and TRANSLATE Evaluations}

The average before- and after-intervention screening rates for the 23 practices can be found in Table 2. One-way repeated measures ANOVA revealed that the difference between mean screening rates before and after the intervention was statistically significant for breast cancer $(36.96 \%$ vs $49.96 \%$; $P=.001)$ and CRC (32.74\% vs $38.30 \% ; P=.001)$. There was a wide degree of variation in the cancer screening rates across practices, with standard deviations ranging from roughly 16 to 21 percentage points. The cancer screening rates reported by the practices participating in this project fall, on average, well below the estimated 2014 New York state rates of $68.1 \%$ for CRC, $78.6 \%$ for breast cancer, and $82.6 \%$ for cervical cancer (estimates are based on self-reported data). ${ }^{28}$ It is important to note, though, that a small number of practices did meet or exceed the statewide screening rates for each cancer. Individual practice screening rates are not listed here but are available upon request.

Table 3 presents the average TRANSLATE model scores before and after the intervention for the 23 practices. The practices, on average, improved in each of the 9 elements measured under 
Table 1. Characteristics of 23 Primary Care Practices Enrolled in the Practice Facilitation and Academic Detailing Project, 2014-2015

\begin{tabular}{|c|c|c|c|}
\hline Practice Structure & Physicians (n) & NPs and/or PAs (n) & ADS Attendees (n) \\
\hline FQHC & $6-15$ & $\geq 2$ & 7 \\
\hline FQHC & $2-5$ & $\geq 2$ & 9 \\
\hline FQHC & $6-15$ & $\geq 2$ & 10 \\
\hline FQHC & $6-15$ & $\geq 2$ & 12 \\
\hline Medical group/health system & $2-5$ & $\geq 2$ & 9 \\
\hline Medical group/health system & 1 & $\geq 2$ & 5 \\
\hline Medical group/health system & 1 & 0 & 5 \\
\hline Medical group/health system & $2-5$ & 0 & 6 \\
\hline Medical group/health system & 1 & 1 & 2 \\
\hline Medical group/health system & $2-5$ & 1 & 17 \\
\hline Medical group/health system & $6-15$ & 1 & 20 \\
\hline Medical group/health system & $2-5$ & 1 & 8 \\
\hline Medical group/health system & $2-5$ & 1 & 12 \\
\hline Medical group/health system & $2-5$ & 1 & 9 \\
\hline Non-profit clinic & $2-5$ & $\geq 2$ & 6 \\
\hline Physician-owned & $2-5$ & $\geq 2$ & 4 \\
\hline Physician-owned & $2-5$ & $\geq 2$ & 5 \\
\hline Physician-owned & $2-5$ & $\geq 2$ & 12 \\
\hline Physician-owned & $6-15$ & $\geq 2$ & 8 \\
\hline University hospital/clinic & $2-5$ & $\geq 2$ & 23 \\
\hline University hospital/clinic & $\geq 16$ & 1 & 9 \\
\hline University hospital/clinic & $6-15$ & 0 & 6 \\
\hline University hospital/clinic & $2-5$ & $\geq 2$ & 6 \\
\hline
\end{tabular}

ADS, academic detailing session; FQHC, federally qualified health center; NP, nurse practitioner; PA, physician assistant.

the TRANSLATE model. However, it is important to note that, individually, some practices did not make measurable improvements across the 2 measurement periods. One-way mixed ANOVA showed statistically significant improvement in scores for the following elements: setting an improvement target, gaining administrative buy-in, using network information systems, conducting audit and feedback activities, and providing staff education, as well as the overall TRANSLATE score for the practices. Two elements were af- fected by PF assignments: 1 PF rated practices with a lower score for target, and another PF rated practices with a higher score for education, compared with other PF practice groups. These differences may have been the result of subjective evaluation differences between the PFs or because of the characteristics of the practices evaluated. Spearman correlation results indicated that higher practice scores in site coordinator engagement and staff education were associated with higher cancer screening rates for all 3 cancer

Table 2. One-Way Repeated Measures Analysis of Variance Comparison of Intervention Cancer Screening Rates Before and After the Intervention at 23 Primary Care Practices Enrolled in the Practice Facilitation and Academic Detailing Project, 2014-2015

\begin{tabular}{lccc}
\hline Cancer Target & $\begin{array}{c}\text { Screening Rate Before the Project, } \\
\text { Mean (SD) }\end{array}$ & $\begin{array}{c}\text { Screening Rate After the Project, } \\
\text { Mean (SD) }\end{array}$ & F Statistic $(P$ Value) \\
\hline Breast cancer & $36.96 \%(17.44 \%)$ & $49.96 \%(20.62 \%)$ & $14.17(0.001)$ \\
Cervical cancer & $35.65 \%(18.68 \%)$ & $38.85 \%(20.34 \%)$ & $0.998(\mathrm{NS})$ \\
Colorectal cancer & $32.74 \%(16.18 \%)$ & $38.30 \%(20.80 \%)$ & $15.740(0.001)$ \\
\hline
\end{tabular}

NS, not significant at $\alpha=.05 ; \mathrm{SD}$, standard deviation. 
Table 3. Comparison of Average Pre- and Post-Intervention TRANSLATE Model Element Scores at 23 Primary Care Practices Enrolled in Practice Facilitation and Academic Detailing Project, 2014-2015

\begin{tabular}{|c|c|c|c|}
\hline \multirow[b]{2}{*}{ TRANSLATE Element* } & \multicolumn{2}{|c|}{ Score, Mean $(\mathrm{SD})^{\dagger}$} & \multirow[b]{2}{*}{$P$ Value } \\
\hline & Before the Intervention & After the Intervention & \\
\hline Target & $2.70(0.95)$ & $3.00(1.02)$ & .031 \\
\hline PF1 & $3.00(1.00)$ & $3.00(1.00)$ & \\
\hline $\mathrm{PF} 2$ & $3.33(0.68)$ & $3.33(0.68)$ & \\
\hline PF3 & $2.80(0.84)$ & $4.00(0.00)$ & \\
\hline $\mathrm{PF} 4$ & $1.86(0.69)$ & $2.00(0.82)$ & .001 \\
\hline Registry and reminder systems & $3.22(0.72)$ & $3.39(0.48)$ & NS \\
\hline Administrative buy-in & $3.15(0.75)$ & $3.43(0.59)$ & .029 \\
\hline Network information systems & $3.22(0.86)$ & $3.48(0.70)$ & .007 \\
\hline Site coordination & $3.09(0.60)$ & $3.22(0.60)$ & NS \\
\hline Local physician champion & $2.74(1.09)$ & $2.80(1.02)$ & NS \\
\hline Audit and feedback & $2.63(0.96)$ & $2.85(0.85)$ & .022 \\
\hline Team approach & $2.85(0.98)$ & $3.11(1.03)$ & NS \\
\hline Education & $2.70(0.67)$ & $2.91(0.63)$ & .057 \\
\hline PF1 & $2.60(0.22)$ & $2.60(0.22)$ & \\
\hline $\mathrm{PF} 2$ & $2.17(0.41)$ & $3.00(0.00)$ & \\
\hline PF3 & $3.60(0.55)$ & $3.60(0.55)$ & .014 \\
\hline $\mathrm{PF} 4$ & $2.57(0.54)$ & $2.57(0.79)$ & \\
\hline Cumulative score & $26.28(3.68)$ & $28.20(3.56)$ & $\leq .001$ \\
\hline
\end{tabular}

*Practice facilitator (PF) scores are displayed only for those measures with significant differences between groups (Bonferonni post-hoc analysis).

${ }^{\dagger}$ Scores are based on evaluations by PFs on a scale of 1 (no accomplishment) to 4 (high accomplishment).

NS, not significant at $\alpha=.05$; SD, standard deviation.

targets. In addition, a team approach was positively correlated with cervical cancer screening rates, and administrative buy-in and audit and feedback were positively correlated with CRC screening rates. Results of the correlation analysis are shown in Table 4.

\section{Staff Surveys and Focus Groups}

A total of 144 individuals responded to the staff surveys; of these, only 56 respondents had linked before/after data from the 23 practices. The majority of the nonresponse occurred during the data collection period after the project because of staff

Table 4. Spearman Correlation of Screening Rates and Post-Intervention TRANSLATE Element Scores After the Intervention at 23 Primary Care Practices Enrolled in the Practice Facilitation and Academic Detailing Project, 2014-2015

\begin{tabular}{lccc}
\hline & & Correlation Coefficient $(P$ Value $)$ \\
\cline { 2 - 4 } & Breast Cancer Screening & Cervical Cancer Screening & Colorectal Cancer Screening \\
\hline Target & $-0.01(\mathrm{NS})$ & $0.01(\mathrm{NS})$ & $0.16(\mathrm{NS})$ \\
Registry and reminder systems & $-0.11(\mathrm{NS})$ & $-0.07(\mathrm{NS})$ & 0.09 (NS) \\
Administrative buy-in & $0.07(\mathrm{NS})$ & $0.20(\mathrm{NS})$ & $0.47(0.024)$ \\
Network information systems & $0.11(\mathrm{NS})$ & $0.11(\mathrm{NS})$ & 0.23 (NS) \\
Site coordination & $0.47(0.023)$ & $0.72(0.001)$ & $0.37(0.086)$ \\
Local physician champion & $-0.06(\mathrm{NS})$ & $-0.04(\mathrm{NS})$ & -0.19 (NS) \\
Audit and feedback & $0.28(\mathrm{NS})$ & $0.31(\mathrm{NS})$ & $0.48(0.022)$ \\
Team approach & $0.20(\mathrm{NS})$ & $0.50(0.025)$ & $0.05(\mathrm{NS})$ \\
Education & $0.42(0.048)$ & $0.54(0.014)$ & $0.48(0.021)$ \\
Cumulative score & $0.28(\mathrm{NS})$ & $0.49(0.030)$ & 0.34 (NS) \\
\hline
\end{tabular}

NS, not significant at $\alpha=.05$. 
Table 5. Common Barriers and Supports for Increasing Cancer Screening Expressed During Focus Groups and Key Informant Interviews at 23 Primary Care Practices Enrolled in the Practice Facilitation and Academic Detailing Project, 2014-2015

\begin{tabular}{|c|c|c|}
\hline & Barriers to Increased Screening & Supports of Increased Screening \\
\hline Patient level & $\begin{array}{l}\text { - Transportation } \\
\text { - Insurance/financial constraints } \\
\text { - Language/communication issues at the } \\
\text { point of care } \\
\text { - Comprehension } \\
\text { - Refusal/noncompliance }\end{array}$ & $\begin{array}{l}\text { - Education and outreach } \\
\text { - Case management and follow up } \\
\text { - Lifestyle-amenable screening methods } \\
\text { - Reduction of structural barriers }\end{array}$ \\
\hline Staff level & $\begin{array}{l}\text { - Lack of time } \\
\text { - EHR data errors } \\
\text { - Lack of investment in quality } \\
\text { improvement interventions }\end{array}$ & $\begin{array}{l}\text { - Shared responsibility to discuss and } \\
\text { document screening with patients } \\
\text { - Standardized data entry and/or EHR } \\
\text { technical assistance } \\
\text { - Performance assessment and feedback } \\
\text { - Point-of-care reminders }\end{array}$ \\
\hline Practice level & $\begin{array}{l}\text { - Lack of personnel } \\
\text { - Workflow inefficiencies } \\
\text { - EHR data errors and reporting limitations } \\
\text { - Two-way communication with specialists }\end{array}$ & $\begin{array}{l}\text { - Quality improvement coaching } \\
\text { - Workflow assessment and adjustment } \\
\text { - EHR "workarounds" } \\
\text { - PCMH certification requirements } \\
\text { - EHR technical assistance }\end{array}$ \\
\hline
\end{tabular}

EHR, electronic health record; PCMH, patient-centered medical home.

turnover and absence at several of the participating practices. The majority of survey respondents were physicians; other respondents included nurse practitioners, physician assistants, registered nurses, licensed practical nurses, and practice/clinic managers. Comparisons of mean responses to Likert-scale questions were conducted only among the 56 linked before/after surveys; most of these differences were not statistically significant. The most important patient-related and system-related barriers to increasing cancer screening as perceived by practice staff centered on a lack of follow-through by patients on screening recommendations and the inability to track patients receiving services at outside offices. Respondents identified the lack of personnel support to both maintain and use registries as among the top barriers to using EHR-based patient registries, and they highlighted provider reminder systems, patient education, and patient reminders as the top QI strategies that would most benefit their practices' ability to increase cancer screening.

Focus groups were only conducted in 3 practices, with 3 to 5 individuals participating in each group (a total of 13 participants). Because of scheduling conflicts, key informants were interviewed at the remaining 20 practices. Participants in the 3 focus groups included practice medical directors $(\mathrm{n}=3)$, practice managers $(\mathrm{n}=3)$, care coordinators $(\mathrm{n}=2)$, and clinical providers $(\mathrm{n}=5)$. Key informants were primarily practice managers, practice QI specialists, and practice medical directors.

Content analysis of the focus group and key informant interview transcripts identified several themes related to cancer screening, which fall broadly within the overarching concepts of barriers to increasing cancer screening and supports for increasing cancer screening at the patient, provider, and practice levels. Table 5 displays a breakdown of these concepts. Other common themes that emerged from analysis focused on the working relationship practices had with the PFs, as well as dynamics within practices that supported systemslevel change.

Consistent engagement by the PFs with their site coordinators helped practices keep a focus on QI initiatives, and those practices that experienced the greatest change in both cancer screening rates and policies had fully engaged staff at several levels within the practice. The ADS oriented practice staff to the goals and targets of the project, increasing buy-in for interventions developed later with the $\mathrm{PF}$. Introducing regular provider performance assessment and feedback mechanisms and increas- 
ing point-of-care reminders and prompts for cancer screening helped clinic staff become more invested in QI projects.

Personnel and funding limitations necessitated the implementation of QI project tasks within existing practice structures and priorities, and workflow assessments guided by PFs were viewed as a valuable tool for achieving this end. Several practices chose to overlap project improvement activities with existing requirements and priorities, including patient-centered medical home and meaningful use targets. PFs were also viewed as a valuable resource for connecting practices with patient outreach resources and community services, including mobile mammography providers and transportation services.

All the practices felt that EHR-based reports measuring patient screening rates did not represent accurate data, but rather reflected only those screening tests that were recorded as structured data within their EHRs. PFs were able to provide guidance to practices on altering data entry procedures and support for data mining, data correction, and chart auditing to improve the validity and reliability of EHR registry data used by the practices.

\section{Discussion}

The outcomes from this QI project suggest that relatively brief practice facilitation combined with academic detailing may be a useful tool to assist primary care practices in the implementation and augmentation of organizational procedures that lead to increased breast cancer, cervical cancer, and/or CRC screening. However, barriers to increased screening remain at the patient, staff, and organizational levels.

Several factors may have contributed to the low screening rates reported by the participating practices. The practices targeted for participation in this project are considered safety-net practices and provide health care services to underserved populations; the low screening rates reported by our practices may be a reflection of the difficulties experienced by their patients in receiving appropriate preventive care. In addition, all the participating practices felt that EHR-based reports likely underreported the true number of patients receiving appropriate screening because of data storage and retrieval issues.

Participation in this project was beneficial to practices to the extent that it initiated the imple- mentation of evidence-based strategies to increase engagement with patients and interventions resulting in sustainable change, including team-based adaptations to office workflows and policies. Rapidcycle improvements, guided by the project PFs, helped practices make changes to improve screening rates that were tailored to their specific priorities and constraints. Our results further indicate that engagement of the practice site coordinator and provision of educational opportunities for practice staff are particularly important to QI intervention success.

Participation in this project encouraged the initiation of data-mapping activities and efforts to address EHR data recording issues. While participants recognized the potential of EHR-based patient registries to help track and increase patient cancer screening, their current systems and staffing constraints reduced the utility of this tool. The initiation of data entry protocols and data correction procedures laid a foundation for the long-term goals of using reliable and valid clinical data to guide patient care and increasing cancer screening at these practices.

\section{Limitations}

Many of the activities initiated during the practice facilitation period will take a substantial amount of time and additional resources to complete, and their impact could not be fully evaluated during the 6-month project period. In addition, the evaluation period may have been too short to capture increased screening for breast cancer and CRC, which often experience a lag between referral and test completion in certain service areas where there are long waiting lists or scheduling delays for specialist providers.

The measurement of practice-level screening rates may have been artificially inflated or underestimated based on how practices defined their active patient lists as well as the guidelines used to define screening populations (USPSTF vs American Cancer Society). However, we believe the information provided by the practices is the most accurate picture available of the screening rates they use to guide practice initiatives and patient care, and thus are the most applicable to the aims of the project. The project did not have access to patient-level or provider-level data within the enrolled practices and thus was only able to assess changes in patient screening status for practices as a whole. 
The TRANSLATE rubric completed by PFs is a subjective evaluation tool. PFs were provided with training at the initiation of the project to enhance the standardization of the evaluations, but variations between PF perceptions of practice systems may have introduced bias to our findings.

\section{Conclusions}

Combining practice facilitation and academic detailing is 1 method through which larger organizations (such as state departments of health) can help practices achieve systems-level changes that enable them to better manage patient population health. An important component of these changes is the optimization of EHR systems: for EHR registry systems to be utilized as tools for improving quality, the data reported must be reliable and valid. ${ }^{29}$ The success of projects using this or other, similar methods is also dependent on the provision of adequate time and resources to initiate QI interventions, as well as interest, investment, and dedication to QI by the staff of participating practices.

\section{References}

1. Gonzalez P, Castaneda SF, Mills PJ, Talavera GA, Elder JP, Gallo LC. Determinants of breast, cervical and colorectal cancer screening adherence in Mexican American women. J Community Health 2012; 37:421-33.

2. U.S. Preventive Services Task Force. Published recommendations. July 2016. Available from: http:// www.uspreventiveservicestaskforce.org/BrowseRec/ Index. Accessed July 13, 2016.

3. NCI Staff. Screening rates for several cancers miss their targets. Cancer Currents blog. May 26, 2015. Available from: http://www.cancer.gov/news-events/ cancer-currents-blog/2015/screening-targets. Accessed January 21, 2016.

4. Gupta S, Sussman DA, Doubeni CA, et al. Challenges and possible solutions to colorectal cancer screening for the underserved. J Natl Cancer Inst 2014;106:dju032.

5. Tammana VS, Laiyemo AO. Colorectal cancer disparities: issues, controversies and solutions. World J Gastroenterol 2014;20:869-76.

6. Wernli KJ, Hubbard RA, Johnson E, et al. Patterns of colorectal cancer screening uptake in newly eligible men and women. Cancer Epidemiol Biomarkers Prev 2014;23:1230-7.

7. Aboagye JK, Kaiser HE, Hayanga AJ. Rural-urban differences in access to specialist providers of colorectal cancer care in the United States: a physician workforce issue. JAMA Surg 2014;149:537-43.
8. Miranda-Diaz C, Betancourt E, Ruiz-Candelaria Y, Hunter-Mellado R. Barriers for compliance with breast, colorectal, and cervical screening cancer tests among Hispanic patients. Int J Environ Res Public Health 2015;13:E21.

9. Singh G, Williams S, Siahpush M, Mulhollen A. Socioeconomic, rural-urban, and racial inequalities in US cancer mortality: part I - all cancers and lung cancer and part II - colorectal, prostate, breast and cervical cancers. J Cancer Epidemiol 2011;2011:107497.

10. Klabunde C, Vernon S, Nadel M, Breen N, Seeff L, Brown M. Barriers to colorectal cancer screening: a comparison of reports from primary care physicians and average-risk adults. Med Care 2005;43:939-44.

11. Anderson BL, Pearlman M, Griffin J, Schulkin J. Conflicting and changing breast cancer screening recommendations: survey study of a national sample of ob-gyns after the release of the 2009 USPSTF guidelines. J Healthc Qual 2013;35:25-35.

12. Zitzelsberger L, Grunfeld E, Graham ID. Family physicians' perspectives on practice guidelines related to cancer control. BMC Fam Pract 2004;5:25.

13. Zhao Y, Goist M. Knowledge of cervical cancer screening guidelines among residents at the Ohio State University Wexner Medical Center. Obstet Gynecol 2014;123(Suppl):94S.

14. Mold JW, Fox C, Wisniewski A, et al. Implementing asthma guidelines using practice facilitation and local learning collaboratives: a randomized controlled trial. Ann Fam Med 2014;12:233-40.

15. Meropol SB, Schiltz NK, Sattar A, et al. Practicetailored facilitation to improve pediatric preventive care delivery: a randomized trial. Pediatrics 2014; 133:e1664-75.

16. Baskerville N, Liddy C, Hogg W. Systematic review and meta-analysis of practice facilitation within primary care settings. Ann Fam Med 2012; 10:63-74.

17. Duckett A, Cuoco T, Pride P, et al. Academic detailing to teach aging and geriatrics. Gerontol Geriatr Educ 2015;36:331-42.

18. O'Brien M, Rogers S, Jamtvedt G, et al. Educational outreach visits: effects on professional practice and health care outcomes. Cochrane Database Syst Rev 2007;4:CD000409.

19. Academic detailing: frequently asked questions. Atlanta: Centers for Disease Control and Prevention; 2014. Available from: http://www.cdc.gov/tobacco/ quit_smoking/cessation/pdfs/academic-detailingfaq508.pdf. Accessed July 13, 2016.

20. Mold JW, Aspy CA, Nagykaldi Z. Implementation of evidence-based preventive services delivery processes in primary care: an Oklahoma Physicians Resource/Research Network (OKPRN) study. J Am Board Fam Med 2008;21:334-44.

21. Community Preventive Services Task Force. Updated recommendations for client- and provideroriented interventions to increase breast, cervical 
and colorectal cancer screening. Am J Prev Med 2012;43:760-4.

22. National survey of primary care physicians' recommendations and practice for breast, cervical, colorectal \& lung cancer screening. Washington, DC: National Cancer Institute, Division of Cancer Control \& Population Sciences. Updated March 2, 2015. Available from: http://healthcaredelivery.cancer.gov/screening rp/. Accessed July 13, 2016.

23. National surveys of colorectal cancer screening policies \& practices. Washington, DC: National Cancer Institute. Updated December 8, 2015. Available from: http://healthcaredelivery.cancer.gov/crc_surveys/. Accessed July 13, 2016.

24. Houser SH, Colquitt S, Clements K, Hart-Hester S. The impact of electronic health record usage on cancer registry systems in Alabama. Perspect Health Inf Manag 2012;9:1f.

25. Quality improvement \& performance management survey. Okemos, MI; Michigan Department of Community Health, Public Health Administration; 2012. Available from: http://www.astho.org/Quality-Improvement/ Toolkit/Michigan-Department-of-Community-HealthQuality-Improvement-and-Performance-ManagementSurvey/. Accessed July 13, 2016.
26. Fox C, Vest B, Kahn L, et al. Improving evidencebased primary care for chronic kidney disease: study protocol for a cluster randomized control trial for translating evidence into practice (TRANSLATE CKD). Implement Sci 2013;8:88.

27. Peterson K, Radosevich D, O'Connor P, et al. Improving diabetes care in practice: findings from the TRANSLATE trial. Diabetes Care 2008;31: $2238-43$.

28. State cancer profiles. Quick profiles: New York. Washington, DC: National Cancer Institute; 2016. Available from: https://statecancerprofiles.cancer.gov/ quick-profiles/index.php? statename $=$ newyork\# $\mathrm{t}=1$. Accessed July 13, 2016.

29. Summary report: use of electronic medical records to facilitate colorectal cancer screening in community health centers. Prepared for: National Colorectal Cancer Roundtable, American Cancer Society, Inc., National Association of Community Health Centers. Deerfield, IL; Aeffect Inc.; 2013. Available from: http://nccrt.org/about/disparities/report-on-use-ofelectronic-medical-records-to-facilitate-colorectalcancer-screening-in-community-health-centers/. Accessed July 13, 2016. 